\title{
Die Frequenzabhängigkeit der longitudinalen Protonenrelaxationszeit von Benzol an Natriumchlorid
}

\author{
D. Michel, H. Pfeifer und B. Todt \\ Physikalisches Institut der Karl-Marx-Universität Leipzig, Abteilung Elektronik
}

(Z. Naturforsch. 23 a, 823-826 [1968] ; eingegangen am 6. März 1968)

\begin{abstract}
By means of NMR- and EPR-spectroscopy we investigated the system benzene-sodium chloride. Using spin-echo techniques the proton relaxation time $T_{1}$ of the sorbed benzene has been measured as a function of proton resonance frequency in the interval between $10 \mathrm{kHz}$ and $24 \mathrm{MHz}$ for different temperatures $\left(7^{\circ} \mathrm{C}, 25^{\circ} \mathrm{C}, 50^{\circ} \mathrm{C}\right)$. The benzene content was $0.16 \mathrm{~g} /(\mathrm{g}$ adsorbent) on ground and dried sodium chloride with a specific surface area of $0.1 \mathrm{~m}^{2} / \mathrm{g}$. The two dispersion regions near frequencies of $\omega_{\mathrm{I}} / 2 \pi=24 \mathrm{MHz}$ and $\omega_{\mathrm{I}} / 2 \pi=460 \mathrm{kHz}$ can be explained by magnetic proton-electron- and magnetic proton-proton-interaction respectively. In agreement with EPR-measurements the high-frequency step gives a transverse electron spin relaxation time $\tau \mathrm{S} 2=1.1 \cdot 10^{-11} \mathrm{~s}$ of the paramagnetic impurities, while the relatively long correlation time $\tau_{\mathrm{CA}}=3.5 \cdot 10^{-7} \mathrm{~s}$ from the low-frequency step can be connected with the stochastic reorientation of the benzene molecules adsorbed on active centers. A rough estimation of the activation energy for $\tau_{\mathrm{CA}}$ yields a value of $1.4 \ldots 2.7 \mathrm{kcal} / \mathrm{mole}$.
\end{abstract}

Kernmagnetische Relaxationszeiten hängen von der magnetischen Wechselwirkungsenergie zwischen den Spinmomenten und der stochastischen Bewegung der untersuchten Moleküle ab, die durch die Korrelationszeiten charakterisiert wird. Die getrennte Bestimmung dieser beiden Faktoren, die für die Deutung des Kernspinrelaxationsverhaltens notwendig ist, wird ermöglicht, wenn man die experimentellen Bedingungen 1. durch Temperaturveränderung (Variation der Korrelationszeit $\tau$ ) oder 2. durch die Veränderung der Magnetfeldstärke (Variation der Resonanzfrequenz $\omega$ ) so wählt, daß das Produkt $\omega \tau$ in die Größenordnung von eins kommt. Der Vorzug der zweiten Methode besteht darin, daß dabei nur die Zeeman-Aufspaltung der Kernspinenergieniveaus verändert wird und die mikrodynamischen Parameter des untersuchten Systems selbst ungeändert bleiben.

In den meisten bisher erschienenen Arbeiten zur kernmagnetischen Relaxation adsorbierter Moleküle wird die Temperaturabhängigkeit der Kernspinrelaxation untersucht ${ }^{1-6}$, und nur in geringem Maße findet die zweite Methode Verwendung ${ }^{7-9}$. Zur Untersuchung der Feldstärkeabhängigkeit der longi-

1 D. E. Woessner u. J. R. Zimmerman, J. phys. Chem. 67, 1590 [1963].

2 D. E. Woessner, J. chem. Phys. 39, 2783 [1963].

3 D. E. Woessner, J. phys. Chem. 70, 1217 [1966].

4 H. Pfeifer, F. Przyborowski, W. Schirmer u. H. Stach, Z. phys. Chem. Leipzig 236, 345 [1967].

5 D. Michel, Z. Naturforsch. 22 a, 1751 [1967].

6 D. Michel, Z. Naturforsch. 23 a, 339 [1968].

7 R. J.S. Brown, Bull. Amer. Phys. Soc. 3, 23 [1958]. J. Korringa, D. O. Seevers u. H. C. Torrey, Phys. Rev. 99, 559 [1955]. tudinalen Protonenrelaxationszeit wurde in der vorliegenden Arbeit ein System gewählt (NaCl-Benzol), das infolge der geringen Konzentration an paramagnetischen Verunreinigungen und dem Fehlen einer ausgeprägten Porenstruktur physikalisch relativ übersichtliche Verhältnisse aufweist und damit als Grundlage für weitere systematische Untersuchungen (Variation von Adsorbens und Adsorbat) dienen kann.

\section{Experimentelles}

Das Natriumchlorid ( $\mathrm{NaCl}$ p.a. des VEB Laborchemie Apolda, Reinheitsforderung nach TGL 8502) wurde zunächst 20 Stunden bei $20{ }^{\circ} \mathrm{C}$ vorgetrocknet, danach in einer Porzellan-Kugelmühle gemahlen und anschließend nochmals nach dem gleichen Verfahren getrocknet. Die auf Grund von mikroskopischen Messungen ermittelte spezifische Oberfläche $\left(0,08 \pm 0,03 \mathrm{~m}^{2} / \mathrm{g}\right)$ entspricht im Rahmen der Fehler dem durch Adsorptionsuntersuchungen mit dem ATLAS-BETographen ${ }^{10}$ erhaltenen Wert $\left(0,13 \pm 0,02 \mathrm{~m}^{2} / \mathrm{g}\right)$. Größere spezifische Oberflächen bis zu $30 \ldots 40 \mathrm{~m}^{2} / \mathrm{g}$ könnte man durch Mahlen unter Vakuum oder Stickstoffatmosphäre bei gleichzeitigem Erhitzen ${ }^{11}$ oder durch elektrostatische Fällung von $\mathrm{NaCl}-\mathrm{Dämpfen}{ }^{12}$ erhalten. Für die vorliegenden Untersuchungen reichte jedoch eine spezifische Oberfläche von ca. $0,1 \mathrm{~m}^{2} / \mathrm{g}$ aus. Als Adsorbate fanden Benzol p. a. (vom VEB Berlin-Chemie) und schweres Ben-

8 V. L. Pollak u. R. R. Slater, Chicago APS Meeting 1967.

9 J. Tschernischew, Physikalische Fakultät der Leningrader Shdanow-Universität, private Mitteilung.

10 H. J. Bültemann, Le Vide Paris 17, 201 [1962]. - Für die Ausführung dieser Untersuchungen danken wir Herrn Prof. Dr. Schirmer und Frau Dr. Steinicke, Institut für Physikalische Chemie der DAdW zu Berlin.

11 W. F. Kisenew, Physikalische Fakultät der Moskauer Lomonossow-Universität, private Mitteilung.

12 D. S. Mc Iver u. P. H. Емmetт, J. phys. Chem. 60, 824 [1956]. - K. Heise, Diplomarbeit, Dresden 1960. 
zol mit einem Anreicherungsgrad von 98\%-D (von der Fa. Isocommerz Berlin-Buch) Verwendung, die durch eine wiederholte Anwendung des Einfrieren-AbpumpenAuftauen-Zyklus von gelöstem Sauerstoff befreit wurden. Zur Kontrolle dieses Vorganges dienten Messungen der longitudinalen Relaxationszeiten der freien Flüssigkeiten.

Um ein gutes Signal-Rausch-Verhältnis zu erzielen, wurde eine Beladung von 16 Gew.-Proz. Benzol gewählt bei einer Gesamtmenge von etwa $1 \mathrm{~kg} \mathrm{NaCl}$. Durch ein mehrmaliges Erhitzen und Abkühlen der abgeschmolzenen Proben ließ sich eine inhomogene Verteilung des Adsorbates weitgehend beseitigen. Die Fehler in der Probenbeladung waren nicht größer als 1\%. Gelöstes Wasser (maximal 0,25\%) beeinflußte die Protonenspinrelaxation des Benzols nicht, wie sich aus dem Vergleich mit den Relaxationszeiten von Benzol (bei starken Magnetfeldern) ergab, das vorher mit metallischem $\mathrm{Na}$ trium getrocknet wurde. Für die Messung der Protonenspinrelaxation standen zwei Spin-Echo-Spektrometer zur Verfügung, die in den Arbeiten ${ }^{13-15}$ beschrieben sind. Abb. 1 zeigt die Frequenzabhängigkeit der longitudinalen Relaxationszeit im Bereich zwischen $10 \mathrm{kHz}$ und $24 \mathrm{MHz}$ bei Temperaturen von $7^{\circ}, 25^{\circ}$ und $50^{\circ} \mathrm{C}$. $\mathrm{Da}$ der Einfluß der Relaxationsrate $T_{10}^{-1}$ der freien Flüssigkeit auf die Relaxationsrate $T_{1 \text { eff }}^{-1}$ des Systems nicht vernachlässigt werden kann, ist in Abb. 1 die Differenz $\left(T_{1 \text { eff }}^{-1}-T_{10}^{-1}\right)^{-1}$ mit $T_{10}=14,5 \mathrm{~s} ; 18 \mathrm{~s} ; 25 \mathrm{~s}$ für die angegebenen Temperaturen aufgetragen. Der mittlere Fehler für die einzelnen Relaxationszeiten (Mittelwert aus mindestens 10 Messungen) beträgt im gesamten Frequenzbereich $\pm 4 \%$. Die Temperaturangabe ist

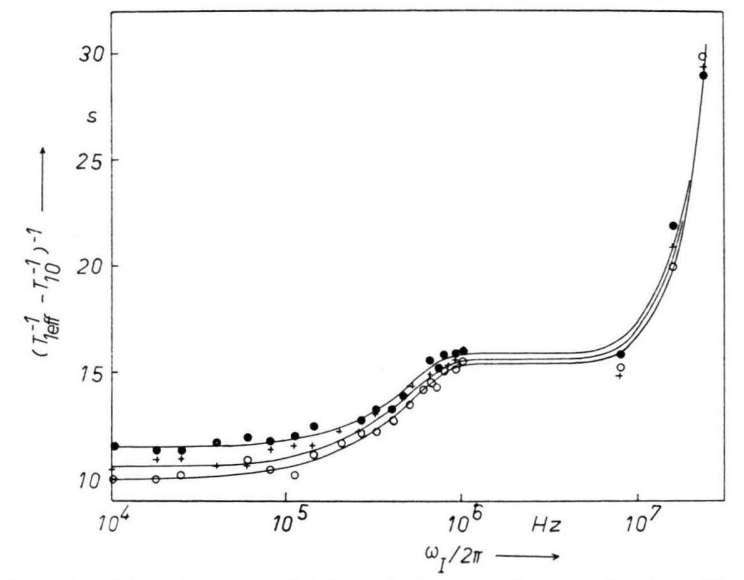

Abb. 1. Die Frequenzabhängigkeit der longitudinalen Protonenrelaxationszeit $\left(T_{1 \text { eff }}^{-1}-T_{10}^{-1}\right)^{-1}$ des Systems Benzol$\mathrm{NaCl}$ bei verschiedenen Temperaturen. $T_{10}$ ist die Relaxationszeit der freien Flüssigkeit. Meßwerte bei $\bullet 50^{\circ} \mathrm{C}$; $+25^{\circ} \mathrm{C}$; $7^{\circ} \mathrm{C}$.

13 H. Sprinz, Wiss. Z. K.-M.-Univ. Leipzig 14, 935 [1965].

14 H. Sprinz, Ann. Phys. Leipzig 20, 168 [1967].

15 H. Pfeifer u. K. H. Weiss, Tagungsband HF-Spektroskopie, Akademieverlag, Berlin 1961.

16 D. Michel, Z. Naturforsch. 21 a, 366 [1966].

17 Herrn Dipl.-Phys. F. Przyвorowski danken wir für die Messungen am JEOL-EPR-Spektrometer JES 3-BQ. auf $1 \%$ genau. Da die kernmagnetischen Relaxationszeiten stark durch paramagnetische Verunreinigungen beeinflußt werden können ${ }^{16}$, wurden auch EPR-Untersuchungen an diesen Systemen durchgeführt ${ }^{17}$. Die in der Tabelle angegebenen Resultate sind Mittelwerte von Messungen an verschiedenen Proben. Es zeigte sich kein Unterschied zwischen der gemahlenen und ungemahlenen bzw. der mit Benzol beladenen und unbeladenen Natriumchloridprobe. Innerhalb der Meßgenauigkeit ist im untersuchten Bereich auch keine Temperaturabhängigkeit des $g$-Faktors, der Elektronenspinrelaxationszeit $\tau_{\mathbb{S} 2}$ und der Intensität der Linie festzustellen. Zur Ermittlung der Elektronenrelaxationszeit $\tau_{\text {S2 }}$ wurde angenommen, daß die EPR-Linie nicht inhomogen verbreitert ist und Lorentz-Form besitzt. Eine genauere Untersuchung der Linienform war infolge des schlechten Signal-Rausch-Verhältnisses nicht möglich.

\section{Auswertung und Diskussion}

Wie man aus dem Kurvenverlauf der Abb. 1 erkennt, treten bei den Frequenzen um $\omega_{\mathrm{I}} / 2 \pi=4,6$ $\cdot 10^{5} \mathrm{~Hz}$ und $\omega_{\mathrm{I}} / 2 \pi=24 \cdot 10^{6} \mathrm{~Hz}$ Dispersionsgebiete auf. Das Verhältnis dieser beiden Frequenzen beträgt 52 und das Verhältnis der Relaxationsraten $\left(T_{1 \text { eff }}^{-1}-T_{10}^{-1}\right)$ vor und nach dem ersten Dispersionsgebiet etwa 1,5 .

Da zwei ausgeprägte Dispersionsgebiete vorliegen, die sich beide durch Funktionen der Form $\left(1+\left(k \omega_{\mathrm{I}}\right)^{2}\right)^{-1}$ beschreiben lassen, entfällt eine Diskussion von Relaxationsmodellen mit einer Verteilung von Korrelationszeiten ${ }^{18}$ und die intermolekulare Wechselwirkung mit kleinen Diffusionssprüngen (vgl. z. B. ${ }^{19}$ ). Wechselwirkungsmechanismen, die nur ein Dispersionsgebiet besitzen, können ebenfalls nicht die einzige Ursache für die gefundene Abhängigkeit sein. Eine Frequenzabhängigkeit mit mehreren Dispersionsgebieten besitzen die Relaxationsraten der dipolaren Proton-Elektron-Wechselwirkung, die Relaxationsraten der dipolaren Proton-Proton-Wechselwirkung mit anisotroper stochastischer Bewegung oder einem Kern- und Molekülaustausch zwischen Bereichen mit unterschiedlicher Beweglichkeit, sowie die Relaxationsraten infolge einer gemischten Proton-Elektron- und Proton-Proton-Wechselwirkung.

\section{a) Proton-Elektron-Dipol-Wechselwirkung}

Wenn die Kristallfeldaufspaltung ${ }^{20}$ und die $\mathrm{Hy}$ perfeinwechselwirkung ${ }^{21,22,14}$ klein gegen die Zee-

18 A. Odajima, Progr. theoret. Phys. Kyoto 10, 142 [1959]. H. A. Resing, J. chem. Phys. 43, 669 [1965].

19 H. E. Heinze u. H. Pfeifer, Z. Phys. 192, 329 [1966].

20 U. Lindner, Ann. Phys. Leipzig 16, 319 [1965].

21 H. Pfeifer, D. Michel, D. Sames u. H. Sprinz, Mol. Phys. 11, 591 [1966].

22 D. Sames u. D. Micher, Ann. Phys. Leipzig 18, 353 [1966]. 
man-Energie sind und das paramagnetische Zentrum einen isotropen $g$-Faktor besitzt, ergibt sich für die longitudinale Protonenrelaxationsrate

$$
T_{1}^{-1}=K_{\mathrm{PE}}\left[\frac{\tau_{1}}{1+\omega_{\mathrm{I}}^{2} \tau_{1}{ }^{2}}+\frac{(7 / 3) \tau_{2}}{1+k_{\nu}{ }^{2} \omega_{\mathrm{I}}^{2} \tau_{1}{ }^{2}}\right]
$$

mit den Beziehungen

und

$$
\begin{gathered}
K_{\mathrm{PE}}=p_{\mathrm{I}} \frac{2}{5} \frac{\gamma_{\mathrm{I}}^{2} g^{2} \mu_{\mathrm{B}}^{2}}{b^{6}} S(S+1), \\
\tau_{l}^{-1}=\tau_{\mathrm{S} l}^{-1}+\tau^{-1}
\end{gathered}
$$

$$
k_{v}=\frac{g \mu_{\mathrm{B}}}{\hbar \gamma_{\mathrm{I}}} \frac{\tau_{2}}{\tau_{1}}
$$

für die Wechselwirkungsenergie $K_{\mathrm{PE}}$, die Korrelationszeiten $\tau_{l}$ und das Verhältnis der Stufenfrequenzen $k_{\nu} . g$ stellt den spektroskopischen Aufspaltungsfaktor, $\mu_{\mathrm{B}}$ das Bohrsche Magneton, $\gamma_{\mathrm{I}}$ das gyromagnetische Verhältnis des Protons, $\omega_{\mathrm{I}}=\gamma_{\mathrm{I}} H_{0}$ die Resonanzfrequenz der Protonen, $p_{\mathrm{I}}$ den Bruchteil der Protonen in der ersten Koordinationssphäre der paramagnetischen Zentren, $b$ den Abstand der Protonen in der ersten Koordinationssphäre vom Zentrum des paramagnetischen Ions, $\tau_{\mathrm{S} l}(l=1,2)$ die Elektronenspinrelaxationszeiten und $\tau$ die thermische Korrelationszeit dar.

Da $g \approx 2$ durch EPR-Messungen gesichert ist (vgl. Tabelle), ergibt sich für das theoretische Verhältnis der Stufenfrequenzen nach Gl. (4) $k_{\nu}=657\left(\tau_{2} / \tau_{1}\right)$, während experimentell ein Wert von $k_{v \exp }=52$ und daraus $\tau_{2} / \tau_{1}=52 / 657=0,079$ folgt. Die theoretische Stufenhöhe am Dispersionsgebiet um $k_{\nu} \omega_{\mathrm{I}} \tau_{1} \approx 1$ beträgt andererseits

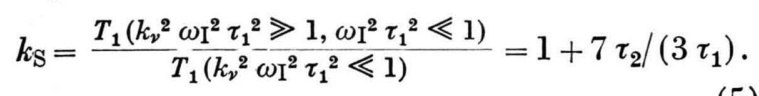

Experimentell bestimmt man aus der ersten Dispersionsstufe bei $460 \mathrm{kHz}$ einen Wert von $k_{\mathrm{S} \text { exp }}=1,5$. Aus diesen Angaben folgt $\left(\tau_{2} / \tau_{1}\right) \approx 0,22$. Neben dieser Diskrepanz, die infolge möglicher systematischer Abweichungen vom theoretischen Verhalten ${ }^{20-23}$ jedoch nicht hinreichen würde, sprechen vor allem die unter Punkt c) zusammengestellten Befunde gegen eine Erklärung allein durch die dipolare ProtonElektron-Wechselwirkung.

23 J. Kärger u. H. Preifer, Zur Theorie der Protonenrelaxation infolge Wechselwirkung mit paramagnetischen Zentren, Ann. Phys., Leipzig, im Druck.

\section{b) Anisotrope stochastische Bewegung der Moleküle}

Falls der Kernverbindungsvektor senkrecht zur Rotationsachse steht, ergibt sich für die effektive longitudinale Protonenrelaxationsrate

$$
T_{1}^{-1}=(1 / 4) T_{1}^{-1}\left(\tau_{\mathrm{CA}}\right)+(3 / 4) T_{1}^{-1}\left(\tau_{\mathrm{CC}}\right),
$$

wobei

$$
\begin{gathered}
T_{1}^{-1}(\tau)=K_{\mathrm{PP}}\left[\frac{\tau}{1+\left(\omega_{\mathrm{I}} \tau\right)^{\varsigma}}+\frac{4 \tau}{1+\left(2 \omega_{\mathrm{I}} \tau\right)^{2}}\right], \\
K_{\mathrm{PP}}=\frac{2}{3} p M_{2}
\end{gathered}
$$

den bekannten Ausdruck für die Protonenrelaxationsrate der Dipolwechselwirkung gleicher Spins darstellt. $M_{2}=2,3 \cdot 10^{9} \mathrm{~s}^{-2}$ bezeichnet das intramolekulare zweite Moment des Benzolmoleküls, $p$ den Bruchteil der Protonen in der ersten Koordinationssphäre der aktiven Zentren und $\tau_{\mathrm{CA}}, \tau_{\mathrm{CC}}$ die Korrelationszeiten der anisotropen Bewegung. Die theoretische Stufenhöhe beträgt $1+\tau_{\mathrm{CA}} \cdot\left(3 \tau_{\mathrm{CC}}\right)^{-1}$, so daß aus dem experimentellen Wert für die Stufenhöhe ein Verhältnis $\tau_{\mathrm{CA}} / \tau_{\mathrm{CC}}=1,5$ folgt. Dieser Ausdruck stimmt nicht mit dem experimentell gefundenen Quotienten der Stufenfrequenzen $\tau_{\mathrm{CA}} / \tau_{\mathrm{CC}}=52$ überein. Die aufgenommene Frequenzabhängigkeit kann somit nicht allein durch eine anisotrope Bewegung gedeutet werden. $\mathrm{Zu}$ der gleichen Schlußfolgerung führt auch eine analoge Betrachtung des Modells „Austausch mit einem Bereich großer Korrelationszeit".

\section{c) Gemischte Proton-Elektron- und Proton-Proton- Wechselwirkung}

Die Temperaturabhängigkeit der Relaxationszeiten am Dispersionsgebiet um Frequenzen von $\omega_{\mathrm{I}} / 2 \pi$ $=24 \mathrm{MHz}$ weist auf eine Proton-Elektron-Wechselwirkung mit der Elektronenrelaxationszeit als Korrelationszeit hin. Diese Deutung wird auch dadurch unterstützt, daß die daraus ermittelte Korrelationszeit gut mit der Elektronenrelaxationszeit $\tau_{\text {§2 }}$ übereinstimmt (vgl. die Tabelle). Experimente mit $\mathrm{C}_{6} \mathrm{H}_{6}$ $\mathrm{C}_{6} \mathrm{D}_{6}$-Gemischen als Adsorbat widersprechen dieser Annahme nicht, da sich bei den hohen Frequenzen die longitudinale Relaxationszeit als unabhängig

\begin{tabular}{cccc}
\hline $\begin{array}{c}\text { Temperatur } \\
\text { in }{ }^{\circ} \mathrm{C}\end{array}$ & $g$-Faktor & $\begin{array}{c}\tau_{\mathrm{S} 2} \\
\text { sec }\end{array}$ & $\begin{array}{c}\text { Intensität } \\
\text { Spins } / g\end{array}$ \\
\hline 7 & 2,1 & $10^{-11}$ & $3,0 \cdot 10^{17}$ \\
25 & 2,1 & $1,1 \cdot 10^{-11}$ & $2,7 \cdot 10^{17}$ \\
50 & 2,1 & $1,1 \cdot 10^{-11}$ & $3,5 \cdot 10^{17}$ \\
\hline
\end{tabular}

Resultate von EPR-Messungen an dem untersuchten System $\mathrm{NaCl}$ (+ Benzol). 
vom H/D-Verhältnis erweist (vgl. ${ }^{6}$ ). Die Größe der Elektronenspinrelaxationszeit zeigt darüber hinaus, daß das zweite Dispersionsgebiet durch die erste Stufe der dipolaren Proton-Elektron-Wechselwirkung oder die skalare Wechselwirkung gegeben wird. (Erst Messungen bei höheren Frequenzen können darüber entscheiden, in welchem Maße die dipolare oder skalare Wechselwirkung vorliegt.)

Da das zweite Dispersionsgebiet um Frequenzen von $24 \mathrm{MHz}$ durch die erste Stufe der dipolaren Proton-Elektron-Wechselwirkung und/oder die skalare Wechselwirkung erklärt wird, bleibt für das erste Dispersionsgebiet $\left(\omega_{\mathrm{I}} / 2 \pi=460 \mathrm{kHz}\right)$ nur noch die Proton-Proton-Wechselwirkung.

Dieser Zuordnung widerspricht auch nicht die stärkere Temperaturabhängigkeit bei niedrigeren Frequenzen (s. u.).

Eine Erklärung des ersten Dispersionsgebietes mit dem Modell der isotropen Rotation ist von der Geometrie des Benzolmoleküls her unwahrscheinlich. Zur Deutung dieses Gebietes benutzen wir daher das Relaxationsmodell der anisotropen Rotation an aktiven Zentren. Die zugehörige Korrelationszeit $\tau_{\mathrm{CA}}$ (vgl. Gl. (6)) ist dann die Umorientierungszeit der sechszähligen Symmetrieachse.

Somit folgt für die effektive Relaxationsrate

$$
T_{1 \mathrm{eff}}^{-1}-T_{10}^{-1}=T_{1 \mathrm{PE}}^{-1}+T_{1 \mathrm{PP}}^{-1} .
$$

Dabei wird $T_{1 \mathrm{PE}}^{-1}$ durch die Beziehung

$$
\begin{aligned}
T_{1 \mathrm{PE}}^{-1} & =\left[K_{\mathrm{PE}}+p_{\mathrm{I}} \frac{2}{7}\left(\frac{A}{\hbar}\right)^{2} S(S+1)\right] \frac{(7 / 3) \tau_{2}}{1+\left(657 \omega_{\mathrm{I}} \tau_{2}\right)^{2}} \\
& =K_{\mathrm{PE}}^{\prime} \frac{(7 / 3) \tau_{2}}{1+\left(657 \omega_{\mathrm{I}} \tau_{2}\right)^{2}}
\end{aligned}
$$

gegeben. $A$ stellt die Kopplungskonstante der skalaren Wechselwirkung dar, für die übrigen Bezeichnungen vgl. man die Gln. (1) bis (4); der erste Summand von Gl. (1) kommt im vorliegenden Frequenzbereich noch nicht zum Tragen und wurde daher vernachlässigt.

Für den Beitrag $T_{1 \mathrm{PP}}^{-1}$ gilt

$T_{1 \mathrm{PP}}^{-1}=K_{\mathrm{PP}}(1 / 4)\left[\frac{\tau_{\mathrm{CA}}}{1+\left(\omega_{\mathrm{I}} \tau_{\mathrm{CA}}\right)^{2}}+\frac{4 \tau_{\mathrm{CA}}}{1+\left(2 \omega_{\mathrm{I}} \tau_{\mathrm{CA}}\right)^{2}}\right]$.

Die verwendeten Symbole sind bei den Gln. (6) bis (8) erklärt; der zweite Summand von Gl. (6) kann hier vernachlässigt werden. Die experimentell aufgenommene Frequenzabhängigkeit wird mit

$$
K_{\mathrm{PE}}^{\prime}=1,75 \cdot 10^{9} \mathrm{~s}^{-2},
$$

$$
\begin{aligned}
K_{\mathrm{PP}} & =6,64 \cdot 10^{4} \mathrm{~s}^{-2}, \\
\tau_{2} & =\tau_{\mathrm{S} 2}=1,1 \cdot 10^{-11} \mathrm{~s}
\end{aligned}
$$

und

$$
\tau_{\mathrm{CA}}=3,5 \cdot 10^{-7} \mathrm{~s}\left(25^{\circ} \mathrm{C}\right)
$$

gut angenähert, wie man aus Abb. 2 erkennt. Aus Gl. (8) ergibt sich mit $K_{\mathrm{PP}}$ nach (13) und dem Wert des zweiten Momentes $\left(2,3 \cdot 10^{9} \mathrm{~s}^{-2}\right)$ ein Verdünnungsfaktor $p=4,33 \cdot 10^{-5}$. Mit der spezifischen Oberfläche $\left(0,1 \mathrm{~m}^{2} / \mathrm{g}\right)$, dem bekannten Flächenbedarf

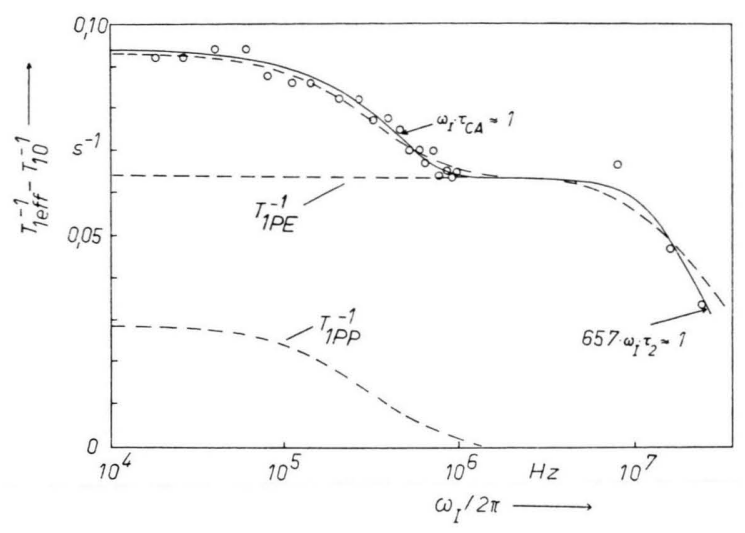

Abb. 2. Vergleich der theoretischen und experimentellen Werte für die Protonenrelaxation des Systems Benzol-NaCl bei $25{ }^{\circ} \mathrm{C}$.

des Benzolmoleküls $\left(42 \AA^{2}\right)$ und der vorliegenden Beladung (16 Gewichtsprozent) folgt daraus, daß jedes fünfte Benzolmolekül der ersten monomolekularen Schicht an einem aktiven Zentrum angelagert ist und die Korrelationszeit $\tau_{\mathrm{CA}}=0,35 \mu$ s für seine Umorientierung besitzt. Wegen des relativ kleinen Temperaturbereiches läßt sich die Aktivierungsenergie aus der Temperaturabhängigkeit nur abschätzen $(1,4 \ldots 2,7 \mathrm{kcal} / \mathrm{Mol})$. Die Korrelationszeit der übrigen Benzolmoleküle in der ersten monomolekularen Schicht kann aus den vorliegenden Messungen nicht bestimmt werden. Da gemäß Gl. (12) $\tau_{2}=\tau_{\text {S2 }}$ gilt, läßt sich lediglich als untere Grenze $10^{-11} \mathrm{~s}$ angeben. Eine Auswertung des Proton-Elektron-Anteils ist nicht möglich, weil in dem Ausdruck für $K_{\mathrm{PE}}^{\prime}$ (vgl. Gln. (2), (10) und (12)) drei unbekannte Größen $\left(p_{\mathrm{I}}, b, A\right)$ enthalten sind. Zwar ist die Gesamtzahl der paramagnetischen Verunreinigungen bekannt (vgl. Tab.), jedoch benötigt man für $p_{\mathrm{I}}$ nur den in der Oberfläche sitzenden Bruchteil, der nicht damit korreliert zu sein braucht ${ }^{5}$. 